\title{
XI International Leprosy Congress, Mexico City 1978
}

\section{(1) WORKSHOP ON EXPERIMENTAL LEPROSY}

\author{
Chairman: C. H. Binford \\ Participants: $\quad$ M. E. Amescua; L. M. Balina; J. C. Convit; S. Innami; W. F. \\ Kirchheimer; G. Klingmueller; K. Kohsaka; K. Nakamura; \\ D. Opromolla; E. E. Storrs; G. P. Walsh; J. Lew-unable \\ to attend; M. J. Colson (representing H. L. Fieldsteel); A. C. \\ McDougall (representing R. J. W. Rees) \\ Observers: J. Delville; Dr Elsler; Dr Makino
}

At the X International Leprosy Congress, 1973, in Bergen, the Committee on Advances in Experimental Leprosy reported the developments in the use of the normal mouse, the thymectomized irradiated mouse, the neonatally thymectomized Lewis rat and the nine-banded armadillo, Dasypus novemcinctus. Also initial results with the Korean chipmunk were reported.

During the past 5 years there has been intense activity in experimental leprosy and several new models were introduced. The members of the workshop who reported on experiments in new animals brought several sets of appropriately stained histopathological sections for study under the 12 microscopes which were generously provided by the Organizing Committee.

\section{EUROPEAN HEDGEHOG (Erinaceus europaeus)}

In Germany, experimental inoculation with Mycobacterium leprae was initiated in the European hedgehog, a small animal (less than $1 \mathrm{~kg}$ ) with a body temperature of 35 to $36^{\circ} \mathrm{C}$ when not in hibernation. This animal is readily adjusted to a laboratory environment and will breed in captivity. In some animals intracutaneous and subcutaneous inoculation with $M$. leprae resulted, after many months, in local lesions that histopathologically showed epithelioid cell granulomatous reactions with small numbers of AFB. Investigators in the United Kingdom, stimulated by the work in Germany, inoculated a group of hedgehogs in footpads and another group intravenously. Two intravenously infected hedgehogs sacrificed 20 months post-inoculation showed widespread dissemination with large numbers of AFB in macrophages. The lepromatous lesions were found in lymph nodes, liver, spleen, tongue, footpads and ears. Intraneural involvement with $\boldsymbol{M}$. leprae was observed in dermal and sciatic nerves.

The members of the Workshop were impressed by the results obtained in these initial experiments with this small animal which is readily available in Europe and easily adjusted to laboratory environment, and recommended that the investigators vigorously continue their experiments. 


\section{NUDE MOUSE}

The nude athymic mouse (BALB/C-nu/nu) was presented by investigators from Tokyo and Osaka as a model for experimental lepromatous leprosy. The mice were maintained under specific pathogen-free conditions. Severe lepromatous lesions were obtained in liver, lung, spleen, nerves, bone marrow, testis, ears, tail and the inoculated foot. Eight months after inoculation, up to $10^{10} \mathrm{M}$. leprae could be recovered from footpads.

The investigator from Tokyo reported enhancement of the development of the lepromatous disease in the nude mouse by inoculating the animal with young thymic cells ( 1 to 2 weeks). The members of the Workshop were convinced that with further study the nude mouse gives promise of becoming a valuable model for immunological investigations and experimental chemotherapy.

\section{NEONATALLY THYMECTOMIZED LEWIS RAT}

An account was given from the U.S.A. of the widespread dissemination of bacilli in this model following the intravenous injection of $10^{7} \mathrm{M}$. leprae 12 months previously. Moreover, in contrast to the mouse footpad in which the maximum inoculum is $10^{4}$ bacilli (more than this being immunogenic), it is possible to inoculate $10^{7}$ bacilli into the footpad of the NTLR and still detect bacillary growth. Heavily infected NTLR are being used in chemotherapy experiments in order to provide information on persistence of $M$. leprae. This information would be useful in the design of therapeutic regimens for patients with multibacillary leprosy. Monitoring of therapy in this experimental model and in human patients is being carried out by inoculation of large numbers of bacilli into the footpads of NTLR.

\section{ARMADILLOS}

The use of the armadillo in leprosy research has advanced rapidly during the past 5 years. For the first time in the history of leprosy, sufficient quantities of $M$. leprae could be made available for basic research in microbiology, immunology and biochemistry. WHO has seized this opportunity by developing an extensive, comprehensive programme in immunology (IMMLEP), utilizing lepromatous tissue of armadillos captured in southern U.S.A.

In May 1977, the Pan American Health Organization conducted at the Instituto Nacional de Dermatologia, Caracas, Venezuela, a 5-day Workshop on the Armadillo as an Experimental Model in Biomedical Research. The report of this Workshop will be made available in December 1978, in PAHO Scientific Publication No. 366 (178 pages).

Stimulated by the Workshop in Caracas, programmes to use armadillos in leprosy research are developing in many countries of the Americas. In addition to $D$. novemcinctus, several other species found only in South America are being investigated. Active programmes utilizing nine-banded armadillos from southern U.S.A. are being carried out in countries where armadillos are not naturally available. 
Reports on progress of leprosy research with armadillos were made by several participants of this Workshop.

\section{Venezuela}

Very encouraging results were reported in the Dasypus sabanicola, the eight-banded armadillo, native to certain regions of Venezuela and Colombia. This animal is much smaller $(1.5 \mathrm{~kg})$ than $D$. novemcinctus and more easily handled in the laboratory. On inoculation with $M$. leprae a very significant number of $D$. sabanicola develop severe lepromatous leprosy, while some animals develop near-tuberculoid lesions, suggesting that this model may be useful in studying the several types of leprosy found in man.

\section{United Kingdom}

The report from the U.K. revealed that it is possible to successfully maintain colonies of the nine-banded armadillo ( $D$. novemcinctus) in a country where this animal is not naturally available. Fifty-six percent of 16 armadillos surviving from a group of 20 which were donated by the Gulf South Research Laboratory, New Iberia, Louisiana, within 2 years after inoculation developed disseminated lepromatous lesions involving major tissues and organs. Dermal nerves were frequently involved. Large nerve involvement was seen in some animals. Histopathologically the lesions were similar to those reported from Carville and New Iberia, La.

\section{Argentina}

In Argentina, 3 species native to the country, Zaedus pichey, Chaetophractus villosus and Dasypus hybridus, were inoculated by intradermal and intracardiac routes. Repeated inoculations were done in some animals. In $Z$. pichey and $C$. villosus, granuloma developed at the sites of inoculation. Some granulomas histopathologically showed tuberculoid changes. The lesions did not advance locally or disseminate. The results of inoculation in $D$. hybridus could not be learned because no animals survived more than 6 months.

\section{Brazil}

A large programme for using armadillos in leprosy research is in progress. After unsuccessful results in several species, the project now is principally concerned with determining the susceptibility of the native $D$. novemcinctus to leprosy. Eight $D$. novemcinctus were found not to be infected after periods of observation up to 18 months. Much longer periods of observation will be used.

\section{Paraguay}

In Paraguay, the inoculations of Chactophractos vellerosus and Euphractus septicinctus were unsuccessful. The first trials with $D$. novemcinctus were interrupted by loss of the colony from an epizootic infection. An extensive programme using principally $D$. novemcinctus is now underway. 


\section{Mexico}

In Mexico, facilities for use of the native $D$. novemcinctus have been provided and 18 animals have now been adapted to the laboratory environment. Basic information on the natural bacterial flora of these armadillos and haematologic profiles have been obtained.

\section{Carville, $L a$}

At Carville, programmes previously reported have been continued and valuable information has been provided on results of massive intravenous inoculation to produce early harvests of lepromatous tissues for basic research.

An interesting report was made on the results obtained from vaccinating armadillos with heat-killed whole $M$. leprae in incomplete Freund's antigen 8 months prior to challenge with viable $M$. leprae. The results in a small group of animals were promising and it is hopeful that further experimental vaccination of armadillos will be done.

\section{New Iberia, $L a$}

Inoculation with $M$. leprae of two Dasypus hybridus obtained from Argentina resulted after 24 months in severe, disseminated lepromatous leprosy in one animal and no infection in the other. Histopathologically, the lesions were indistinguishable from those seen regularly in infected $D$. novemcinctus. The positive result in this animal should provide impetus for effectively using $D$. hybridus in programmes of experimental leprosy in Argentina where this species is readily available.

Experience with the inoculation of approximately $500 \mathrm{D}$. novemcinctus showed that up to $60 \%$ will develop disseminated leprosy at doses of $10^{7}$ to $10^{8}$ $M$. leprae. Histopathological evaluation of tissues from the experimentallyinoculated animals provided experience that later was invaluable in the study of armadillos naturally infected with mycobacteria later identified as M. leprae.

Studies of the tissues and organs from normal newborn and adult armadillos showed the reticuloendothelial system (thymus, spleen, lymph node, tonsils, and Peyer's patches) of $D$. novemcinctus to be well developed and morphologically intact. From these studies it was concluded that the susceptibility of the nine-banded armadillo to leprosy was not a result of malformation of organs or tissues needed to mount an effective immune response.

A shift in programme emphasis from the experimental disease to the naturally acquired disease was made following the discovery in 1974 of indigenous leprosy in wild-caught nine-banded armadillos.

\section{Indigenous leprosy in wild armadillos}

The most recent data on indigenous leprosy in D. novemcinctus captured from the wild were presented. Sixty animals from 11 locations in Louisiana and one location in Texas have been found by the Gulf South Research Institute, New Iberia, La to have lepromatous leprosy. Confirmation of indigenous leprosy in armadillos has been reported by the CDC, Atlanta, the University of Texas, Galveston and the USPHS Hospital, Carville, La. An 
armadillo captured in Texas and kept for 2 years in the zoo at San Diego, California was found to have disseminated lepromatous leprosy.

At New Iberia, the best method for identifying leprosy in a live animal was by examination of slit smears from the base of ears. Lymph nodes were consistently involved but obtaining biopsy specimens of lymph nodes from live animals during a survey study is not practical.

By the criteria proposed at the London Congress (1968) and methods subsequently developed (pyridine extraction; fluorescent antibody methods) the cause of the indigenous disease is indistinguishable from $M$. leprae.

The indigenous leprosy found in D. novemcinctus in the U.S.A. provides an opportunity for comprehensive studies on the epizootiology of this disease and from such studies it is hoped that some answers to problems in the epidemiology of human leprosy will be found.

\section{Reproduction of armadillos in captivity}

A scientist from the Medical Research Institute, Melbourne, Florida, reported current studies in reproduction being carried out in that institute and previous studies carried out at New Iberia. Reproduction was given top priority at the PAHO Workshop in Caracas in 1977 and there is general agreement that the full potential of the armadillo in biomedical research will not be obtained until breeding in captivity can be achieved and laboratory-bred animals provided in numbers sufficient for experimental work.

It was emphasized that the stress of captivity is probably responsible for interrupting the normal ovulation phases of the female. Sperm cells of males in captivity, although lower than from males when captured, appear adequate to fertilize ova of females ovulating regularly. Although artificial stimulation of ovulation is possible, it has not resulted in successful impregnation and gestation. Modification of the environment of captivity to reduce stress may prove to be the solution to reproduction.

At the Zoonosis Paramedical Center (CEPANZO) in Argentina in an area where $D$. hybridus is readily available, facilities have been developed for experimental breeding and it is hoped that successful breeding of D. hybridus in numbers adequate for experimental use will result.

\section{KOREAN CHIPMUNK}

Unfortunately, because of transportation difficulties, Joon Lew MD PhD who was scheduled to present his progress in experiments with the Korean chipmunk, Eutamias sibricus Asiaticus, arrived after the conclusion of the Workshop, but presented his finding to some members of the Workshop. In these animals inoculation of $10^{7} \mathrm{M}$. leprae in footpads resulted in gross nodules appearing 7 to 17 months post-inoculation in 10 to $20 \%$ of the animals. Histopathologically, the lesions were advanced lepromatous. Nerves were distinctly involved. Lymph nodes were massively involved and liver and spleen less involved.

These chipmunks, while readily available in nature so far, have not bred in captivity. Obviously, work with this animal that without immunosuppression develops lepromatous leprosy should continue. 


\section{Conclusion}

This Workshop has provided information on several new models for the study of lepromatous leprosy. During the period since the last Congress, significant progress has been made on the use of the nine-banded armadillo ( $D$. novemcinctus) in leprosy research. At this Workshop, the eight-banded armadillo ( $D$. sabanicola) has also been shown to be a model for lepromatous leprosy and hopefully, for other types of leprosy.

With the opportunities now available for investigators in experimental leprosy, the use of these models in studying the pathogenesis, prevention, epidemiology and treatment of human leprosy appear unlimited.

\section{(2) WORKSHOP ON MICROBIOLOGY}

Chairman: Dr S. Pattyn

Rapporteur: Dr P. Draper

Participants: Dr M. Abe; Dr O. Closs; Dr W. M. Meyers; Dr R. Navalkar; Dr K. Prabhakaran; Dr H. Sansarricq; Dr O. Skinsnes; Dr J. Stanford; Dr N. Morrison; Dr C. C. Shepard

\section{MORPHOLOGY}

Mycobacterium leprae, when stained by the Ziehl-Neelsen procedure, is an acid-alcohol-fast, weakly curved bacillus, 0.3 to $0.4 \mu \mathrm{m} \times 2$ to $7 \mu \mathrm{m}$, sometimes showing a metachromatic granule of unknown nature. In all mycobacteria-rich tissues it is possible to find some non-acid-fast organisms. The significance of these, and particularly the possibility that they may be young forms, should be investigated.

In human lepromatous tissues, leprosy bacilli frequently occur in round to oval clumps called globi, the organisms being situated in cigar-shaped bundles.

Commonly the majority of bacteria stain irregularly. Provided that staining techniques are carefully standardized, there is a strong correlation between regular (solid) staining in smears and viability, measured by the mouse footpad model. Harada (1977) has shown that batches of fuchsin having particular light absorbance characteristics give the most satisfactory staining.

Fisher and Barksdale (1971, 1973) and Convit and Pinardi (1972) reported that the acid-fastness of $M$. leprae was extractable with pyridine, and that this was specific for the species. However, Skinsnes et al. (1975) have shown that pyridine extractability of acid-fastness is a characteristic of aging, non-viable bacilli, and not unique to the leprosy bacillus. Sula et al. (1978) also found that the reaction was not specific.

In the electron microscope, shadowed preparations of $M$. leprae are seen to contain both uniformly dense and beaded forms of the bacilli. After shadowing or negative staining of $M$. leprae suspensions, intertwining paired fibrous structures 10 to $30 \mathrm{~nm}$ in width, forming a network in the walls, can be seen. Nishiura et al. (1969) have described "band-like structures" parallel to the short axis of the cell.

In ultrathin sections, $M$. leprae has a cell wall 15 to $20 \mathrm{~nm}$ thick apparently 
consisting of an inner electron-dense and an outer electron-transparent layer, surrounding the cytoplasmic membrane. Large mesosome-like structures are frequently observed, continuous with the cytoplasmic membrane. In tissues, at least, bacterial division is by transverse fission. There have been numerous reports of spheroplasts and other aberrant forms in leproma-derived material. It is important to establish the nature of these and their relation to leprosy.

\section{BIOCHEMISTRY, METABOLISM AND GROWTH}

The presence of mycolic acids in $M$. leprae was first demonstrated by Etemadi and Convit (1974). The large amounts of $M$. leprae obtainable from armadillo tissue allowed a chemical study of the cell walls (Draper, 1976). Like other actinomycetales, $M$. leprae has in its wall mycolic acids, arabinogalactan and peptidoglycan. The mycolic acids resemble 2 of the 3 found in $M$. tuberculosis, and differ from those found in $M$. avium, $M$. lepraemurium, $M$. vaccae and $M$. smegmatis. The peptidoglycan contains diaminopimelic acid, D-alanine, glutamic acid, glucosamine, muramic acid and substantial amounts of glycine. The simultaneous occurrence of glycine and meso-diaminopimelic acid in bacteria is rare and does not occur in other mycobacteria analysed so far. At the present time this is an important differential characteristic of $M$. leprae.

Knowledge of metabolic processes in M. leprae remains limited. Among enzyme activities reported to occur are dopa oxidase (Prabhakaran, 1967), glutamate decarboxylase (Prabhakaran and Bragança, 1964), $\beta$-glucuronidase and $N$-acetyl- $\beta$-glucosaminidase (Matsuo and Skinsnes, 1974). In each case controversy persists about the origin of the activity, whether it is bacterial or host-derived. More investigation is needed.

The organism is reported to take up, and to incorporate into insoluble products, radiolabelled thymidine and dopa (Ambrose et al., 1978). Uptake is apparently inhibited by dapsone and rifampicin, and by diethyldithiocarbamate in the case of dopa.

The drug dapsone is bacteriostatic for M. leprae in very low concentrations: $\mathrm{MIC}=0.003 \mu \mathrm{g}$ per $\mathrm{ml}$. This high sensitivity is an unusual property.

$M$. leprae can be readily transmitted to animals, normal mice, rats and hamsters (limited infections); armadillos, immunosuppressed mice and rats and congenitally athymic (nu/nu) mice (disseminated infections). The evolution of the infection, particularly in the mouse footpad, is characteristic for $M$. leprae when compared with other mycobacteria. The generation time during the exponential phase of multiplication in the mouse footpad is 11 to 13 days. Isolates of $M$. leprae in mouse footpads vary slightly in only two properties: the average rate of growth between inoculation and harvest, and the number of bacilli in the harvest.

\section{ANTIGENIC STRUCTURE}

Abe et al. $(1970,1972)$ found 2 antigens in human lepromatous nodules: a protein antigen thought to be specific for $M$. leprae and a polysaccharide antigen common to other mycobacteria. Abe et al. (1976) has also 
demonstrated an insoluble antigen specific for $M$. leprae by indirect immunofluorescence. This has been useful in identifying organisms as $M$. leprae. Navalkar $(1971,1976)$ found 4 antigens common to other mycobacteria and one protein antigen specific for $M$. leprae.

Stanford (1976) was able to detect 12 antigens in disrupted M. leprae from infected armadillo tissue. Six of these were common to all mycobacteria, 4 were apparently species-specific and 2 of uncertain specificity. Closs detected 20 to 30 antigen components in $M$. leprae. None of these has been demonstrated to be specific for $M$. leprae, but at least some of them possess $M$. leprae-specific determinants as part of the molecule.

\section{CULTURE}

Criteria that should be applied to claims of in vitro culture of $M$. leprae are:

(a) The isolation procedure should ideally be successful in a percentage of attempts when bacilli-rich material, derived from untreated humans or experimental animals, is used.

(b) Multiplication should be regular and significant (taking into account experimental errors and possible artifacts of the techniques used).

(c) Ideally it should be shown that the bacilli can be passaged indefinitely.

Attempts to grow $M$. leprae in tissue culture have sometimes indicated limited multiplication but results were irregular. Recently Talwar's group have shown limited but continuous incorporation of thymidine into bacteria derived from human biopsies, cultured in macrophages.

There have been several recent reports on organisms cultivable from human and armadillo leprosy tissues, notably that of Skinsnes et al. (1975). Proof acceptable to all workers that these organisms are identical with $M$. leprae has not yet been obtained. Among criteria that should be used to demonstrate identity are:

(i) All in vitro-grown strains obtained should be identical in most if not all characters, including drug-sensitivity patterns. Strains may differ in a small number of tests (biotypes).

(ii) Strains should be identical with $M$. leprae in antigen components known to contain $M$. leprae-specific determinants.

(iii) The possibility of contamination from the environment should be considered; isolates should differ from currently known mycobacterial species.

(iv) The strains obtained should behave in experimental animals in a similar way to $M$. leprae derived from human tissue, particularly with respect to nerve invasion.

(v) Standardized suspensions of killed organisms prepared from the cultures should give negative Mitsuda reactions in lepromatous forms of the disease and positive reactions in tuberculoid cases.

(vi) The cell-wall skeleton of the cultivated strains would be expected to resemble that of tissue-derived $M$. leprae in its chemical composition. 
(vii) 3,4-Dihydroxyphenylalanine (dopa) oxidase has been reported to be active in $M$. leprae and has been proposed as a specific test. Several workers have attempted to use this test, but with contradictory results. It is important that procedures for preparing bacterial suspensions and for performing the test are standardized, so that its significance may be established.

The greater the number of characteristics in which the proposed isolate differs from $M$. leprae, the greater the possibility that it is, in fact, a contaminant. Up to the present no cultures have been shown to satisfy all the above criteria, though not all have been fully tested.

\section{Recommendations}

(a) In order to accelerate progress towards in vitro cultivation of M. leprae, the setting up of multidisciplinary teams of investigators as recommended, so that the most recent progress in such areas as cell physiology and molecular biology is applied to the problem. Studies on electron-microscope histochemistry, incorporation of radioisotopes, DNA homology and other genetic investigations and characterization of cell components should be stimulated.

(b) Detailed antigenic characterization of $M$. leprae should be pursued to determine its taxonomic relation with other mycobacteria.

(c) Difficulties have arisen over the use of techniques for determining viability based on morphology in different laboratories, in spite of attempts to ensure that standard methods are used. Additional objective techniques for identif ying viable organisms should be sought.

\section{(3) WORKSHOP ON IMMUNOLOGY OF LEPROSY}

Chairman: W. E. Bullock

Rapporteurs: T. Buchanan; Indira Nath

Participants: $\quad$ M. Abe; R. StC. Barnetson; S. Estrada-Parra; C. K. Job; R. Lai A. Fat; M. Lefford; T. H. Rea; D. S. Ridley; M. Ulrich.

\section{IMMUNOCHEMISTRY AND LEPROSY}

With greater quantities of Mycobacterium leprae made available from infected armadillo tissues during the past 5 years, there have been advances in the characterization of $M$. leprae antigens. There is now evidence that protein antigens specific for $M$. leprae exist.

Specific goals for the coming 5-year period should include studies to confirm this evidence and to characterize putative $M$. leprae-specific antigens with respect to:

(a) The methodology for purification of these antigens.

(b) Defining the physicochemical properties of these antigens that may help to increase their yields from bacilli and inf ected tissues. 
(c) Development of antigen-specific assay systems to allow quantitation either of antibody to these antigens or of antigens present in infected tissues.

(d) Assessment of the role of these antigens in the stimulation of delayedtype hypersensitivity (DTH) and cell-mediated immunity (CMI) specific to $M$. leprae.

(e) Assessment of the role of these antigens in ENL, reversal reactions and the Lucio phenomenon, with emphasis on the search for antigen within lesion sites and within circulating immune complexes.

(f) Determining the significance of antibody to $M$. leprae-specific antigen(s) as an indicator of subclinical leprosy in contacts of lepromatous patients who have positive or negative lepromin skin tests as compared to contacts of tuberculoid patients.

\section{EXPERIMENTAL STUDIES OF IMMUNOREGULATORY MECHANISMS IN LEPROSY}

A significant advance in immunology during the past 5 years has been the identification of functionally specific subpopulations of lymphocytes that modulate the immune response in the mouse. The availability of markers for specific immunoregulatory cell function provides a powerful tool for more precise study of the complex cell-to-cell interactions that result in net help or suppression of the cell-mediated immune defence mechanisms in chronic infectious disease models.

Future studies should first be directed to resolving the nature of the immunoregulatory disturbances evoked by chronic infection of the mouse with Mycobacterium lepraemurium.

Much needed are detailed immunological studies of the normal and $M$. leprae-infected armadillo with special efforts directed to the identification of specific markers for immunoregulatory cell subpopulations in this genus.

Knowledge gained from these models will have direct application to studies of the immunoregulatory disturbances in human leprosy. Critical will be the development of techniques for recognizing specific markers on human regulatory cell subpopulations. As these techniques are perfected, they will yield information of great value in permitting clinical investigators to assess the impact of both chemotherapy and immunotherapy upon the immune functions of leprosy patients; these studies in turn can be expected to aid in the design of therapeutic approaches that will provide maximum long-term benefit.

\section{THE IMMUNOLOGY OF HUMAN LEPROSY}

A complex series of immunological perturbations has been delineated in leprosy patients during the past 5 years. In general, the DTH and CMI responses to contact sensitizing agents and a variety of "recall" antigens, including $M$. leprae, are not impaired substantially in patients with tuberculoid forms of disease. Conversely, in borderline and especially in lepromatous forms of infection, significant impairment of the immune response is observed frequently. Both non-specific generalized impairment of cellular immune 
function occurs as well as a highly specific impairment of $M$. leprae-specific immunity. These abnormalities are frequently associated with disturbances in the ratio of $\mathrm{T}$ and $\mathrm{B}$ lymphocytes in the peripheral blood of lepromatous patients. The non-specific abnormalities of CMI appear to be reversible by chemotherapy of at least several months' duration, whereas impairment of the responses to $M$. leprae antigens is long-lasting.

Additional advances in the immunology of human leprosy include:

(a) the finding that antibodies that appear to be $M$. leprae-specific are detectable in a large percentage of tuberculoid patients as well as in lepromatous patients;

(b) the finding that circulating immune complexes may be present in patients with leprosy, more commonly in those with lepromatous disease; and

(c) evidence that patients with reversal reactions demonstrate increased lymphocyte transformation responses to $M$. leprae.

Goals of high priority during the next 5 years include:

(1) Definition of immunoregulatory subpopulations in man and the assessment of abnormalities within these subpopulations in patients with leprosy.

(2) Extensive studies of macrophage function in leprosy, with emphasis upon the mechanisms of the processing of $M$. leprae antigen.

(3) Longitudinal studies of circulating immune complexes in leprosy patients, and immunopathological studies of tissue reactions evoked by these complexes.

(4) Further exploration of the possibility of a genetic predisposition to leprosy by studying the distribution of HLA antigens in the population of leprosy patients and their families.

(5) Development of a vaccine against leprosy. The availability of M. lepraespecific antigens and of quantitative assays for these antigens, and antibody responses to these antigens, will aid in this pursuit.

(6) Exploration of the thesis that peripheral nerves may serve as "protected sites" for multiplication of $M$. leprae.

\section{(4) WORKSHOP ON EXPERIMENTAL CHEMOTHERAPY}

Chairman:

Rapporteur:

Participants:
Dr R. J. W. Rees

Dr G. A. Ellard

Prof. J. Ambrose; Dr M. J. Colston; Prof. L. Levy; Dr N. E.

Morrison; Prof. S. R. Pattyn; Dr J. M. H. Pearson; Dr J. H. Peters; Prof. J. K. Seydel; Dr C. C. Shepard

Progress in the last 5 years has largely resulted from exploitation of mouse models for the further evaluation of drugs and the demonstration of both drugresistant and persisting drug-sensitive $M$. leprae in patients. 


\section{ANIMAL MODELS}

In view of the continued failure to cultivate $M$. leprae in vitro, the activity of antileprosy drugs must be assessed using animal models. The 2 established animal models are:

\section{(i) Normal mouse}

This, the standard experimental model, enables the minimal inhibitory concentrations (MICs) of drugs against $M$. leprae to be determined, the nature of their antibacterial activity to be assessed, the rate at which viable leprosy bacilli are eliminated in patients during treatment to be measured, and the occurrence of drug-resistant organisms to be detected. Whether drugs have significant antileprosy activity is determined by ascertaining whether continuous administration in the diet prevents multiplication of $M$. leprae in the mouse footpad. Administration of drugs in graded doses allows the minimal effective dose (MED) to be measured. Following the growth of bacilli after administering a drug for a limited period reveals whether or not its activity is purely bacteriostatic (kinetic method), whereas the degree of bacterial killing engendered by drugs displaying potential bactericidal activity can be quantified using the proportional bactericidal test method. The normal mouse can be used to monitor the loss of viability of leprosy bacilli in lepromatous patients early in chemotherapy: when inocula from biopsies are no longer infective at least $99 \%$ of the original viable population must have been killed. The level of drug resistance of strains of $M$. leprae can be determined from their ability to multiply in mice fed graded doses of drugs that are normally inhibitory.

\section{(ii) Thymectomized-irradiated mouse ( $T / R$ mouse)}

This immunologically suppressed mouse enables the killing of $M$. leprae to be followed in treated patients to the time when the proportion of viable bacilli has been reduced to less than $0.1 \%$ of its original value.

\section{PHARMACOKINETICS}

Measurement of serum and tissue levels of drugs in mice fed with the MED enables their MICs to be determined. Pharmacokinetic studies in man indicate by how many fold the peak serum/tissue concentrations are likely to exceed the MIC, the duration after a single dose that inhibitory levels will be maintained, and whether treatment will be actively bactericidal.

\section{CLINICAL TRIALS}

Studies of the treatment of lepromatous leprosy extending from 6 months to 10 years have been monitored in the mouse to assess the rapidity with which viable bacilli are initially killed either by single drugs or by combinations of drugs, and whether drug-sensitive survivors ("persisters") can be eliminated by continued treatment. 


\section{DRUG RESISTANCE SURVEYS}

Because of the importance of dapsone resistance, investigations have been undertaken in various parts of the world to estimate the prevalence of dapsoneresistant strains of $M$. leprae among patients who have relapsed during continuing treatment (secondary resistance) and in those with previously untreated leprosy (primary resistance).

\section{DAPSONE}

The exceptional sensitivity of $M$. leprae to inhibition by dapsone is indicated by its MIC of only $0.003 \mu \mathrm{g} / \mathrm{ml}$. At concentrations near to its MIC, dapsone is essentially bacteriostatic; but at concentrations in excess of 100 times this value it is weakly bactericidal. Studies in both experimental animals and man show that it penetrates readily into all tissues including nerves. A dose of 100 mg dapsone results in peak concentrations that exceed the MIC by a factor of about 500 -fold and maintains inhibitory levels for about 10 days.

Several studies have shown that $30 \%$ or more of leprosy out-patients are grossly irregular in self-administering their dapsone treatment. A reliable method of maintaining inhibitory levels of dapsone is to treat patients with acedapsone, the repository form of dapsone, since $225 \mathrm{mg}$ intramuscular injections of acedapsone maintain dapsone concentrations well in excess of the MIC for over 3 months.

Viable dapsone-sensitive leprosy bacilli ("persisters") can be recovered from up to $50 \%$ of lepromatous patients after as many as 10 years of continuous dapsone monotherapy. Estimates of the prevalence of dapsone-resistant strains of $M$. leprae among lepromatous patients have ranged from $3 \%$ to $20 \%$. In the worst situation new dapsone-resistant cases were occurring at a rate of about $3 \%$ per annum of those at risk. Dapsone-resistance is a stable characteristic of $M$. leprae and resistant strains are clearly infectious for man, since dapsoneresistant strains of $M$. leprae have been isolated from previously untreated patients. Although dapsone is a weak carcinogen in the male rat, epidemiological studies indicate that it is probably not carcinogenic in man. Furthermore neither dapsone nor any of its known metabolites displays mutagenic activity in vitro.

\section{RIFAMPICIN}

The extremely powerful bactericidal activity of rifampicin against $M$. leprae has been demonstrated in the mouse by both the kinetic and proportional bactericidal test methods. Rifampicin is fully active against dapsone-resistant strains, and experimental studies indicate that it penetrates excellently into nerves. In man it reduces the levels of dapsone in the body but this is probably without therapeutic significance. In clinical treatment the bactericidal activity of rifampicin is so powerful that single doses of $1200 \mathrm{mg}$ or as few as 4 consecutive daily doses of $600 \mathrm{mg}$ of the drug killed over $99 \%$ of the viable bacilli. However even 5 years continuous treatment with rifampicin plus thiambutosine failed to eliminate the remaining persisters. Drug-sensitive persisters were also isolated after combined treatment with daily dapsone plus 
rifampicin. Patients have relapsed with rif ampicin-resistant strains of $M$. leprae after treatment with 3 years rifampicin monotherapy, but no such relapses have occurred among patients treated up to 5 years with rifampicin plus thiambutosine.

\section{CLOFAZIMINE}

Because of marked tissue accumulation, it is impossible to determine the MIC of clofazimine against $M$. leprae. Clofazimine treatment is less effective when doses are given at intervals of a week or more. Patients with dapsoneresistant leprosy have been treated for up to 10 years with daily or thrice weekly clofazimine monotherapy without relapses occurring, although the relatively small number of patients treated in this way does not exclude the possibility that clofazimine resistance might eventually emerge in a pattern similar to that originally observed with dapsone. Persisters can also be isolated after many years of continuous clofazimine treatment.

\section{ETHIONAMIDE/PROTHIONAMIDE/THIACETAZONE/ THIAMBUTOSINE}

The most important results of studies of the antileprosy activities of these drugs in the normal mouse and investigations of their pharmacology in man are summarized in Table 1. The corresponding data for rifampicin and dapsone are included for comparison. Strains of $M$. leprae exhibit crossresistance between these drugs, but are not cross-resistant with dapsone.

\section{STREPTOMYCIN/SULPHAMETHOXYPYRIDAZINE}

A pilot clinical trial has shown that the antileprosy activity of streptomycin is inferior to that of dapsone. Experimental and pharmacological data relevant to the antileprosy activity of sulphamethoxypyridazine are given in Table 1.

\section{POTENTIAL NEW DRUGS}

The antileprosy activities of a series of dihydrofolate reductase inhibitors, antithyroid compounds, interf eron inducers, chaulmoogric-, clofazimine- and long-acting rifampicin-analogues have been investigated experimentally. The rifampicin derivatives appeared the most promising for further investigation.

\section{FUTURE STUDIES}

In vitro models

$M$. leprae has been shown to incorporate radioactive thymidine and DOPA in a cell-free system and in human macrophage culture. These systems may provide more rapid means of testing the activity of new drugs and identifying drug-resistant strains.

\section{Animal models}

The neonatally-thymectomized rat and the athymic "nude" mouse may be useful for detecting persisting $M$. leprae and for studies of drug combinations and microbial persistence. A newly-discovered nude rat may also be useful for 
these studies. The armadillo is currently the only source of the large numbers of bacilli required for enzymatic studies of drug action, and represents the only model in which the development of drug resistance can be studied. The pharmacokinetics of dapsone and rifampicin have been studied in the armadillo, but more developmental work will be required before the armadillo can be evaluated as a model for long-term chemotherapeutic studies.

\section{Drug development}

Employing new methods for drug screening, computer-assisted techniques for studies of quantitative structure-action relations, and new analytical methods for studies of enzyme activity, it may be possible to develop new drugs specifically active against $M$. leprae. Employing two experimental models of ENL, it may now be possible to develop an active compound that is non-teratogenic.

\section{Clinical and field studies}

Surveys of the point-prevalence of secondary and primary dapsone resistance must be conducted in various parts of the world. Surveillance programmes should be initiated immediately for rif ampicin resistance, in view of the increasing use of rifampicin, which is often irregular and unsupervised. The efficacy of rifampicin administered intermittently and of ethionamide, prothionamide and thiacetazone must be established in short-term trials monitored by inoculation of normal mice. Combined regimens of drugs in dosage schedules already shown to be effective in short-term monotherapy trials must be tested among patients with lepromatous leprosy in formal clinical trials monitored by inoculation of immunosuppressed rodents. Finally, those combined regimens that appear most promising in formal clinical trials must be tested in the field to determine their acceptability to patients, the ease of their application to leprosy control programmes and, most importantly, their ability to interrupt the transmission of $M$. leprae.

\section{Implications for Present Treatment}

The widespread emergence of dapsone resistance has emphasized the necessity of using combinations of at least two antileprosy drugs for the treatment of lepromatous leprosy. The experimentally determined antileprosy activity and pharmacological characteristics of the available drugs are shown in Table 1. For previously untreated patients, dapsone administered at a dosage of 50 to $100 \mathrm{mg}$ daily must remain the primary drug, and the maintenance of inhibitory levels of dapsone could be guaranteed by the administration of acedapsone in addition to daily doses of dapsone. Of the drugs available for use in combination with dapsone, rif ampicin with its rapid bactericidal activity is the first choice. Clofazimine is less costly than rif ampicin and its antileprosy activity is of the same order as that of dapsone. Thiacetazone might be a suitable drug for inclusion in drug combinations, although the experimental data suggest that one of the thioamides, ethionamide or prothionamide, would be more effective. However, the 
TABLE 1

Minimal inhibitory concentration against M. leprae (MICs), peak serum concentrations, durations of coverage and bactericidal activities of antileprosy drugs

\begin{tabular}{|c|c|c|c|c|c|}
\hline Drug & $\begin{array}{c}\text { MIC } \\
(\mu \mathrm{g} / \mathrm{ml})\end{array}$ & $\begin{array}{c}\text { Dosage } \\
(\mathrm{mg})\end{array}$ & $\begin{array}{c}\text { Ratio* } \\
\text { peak serum } \\
\text { MIC }\end{array}$ & $\begin{array}{c}\text { Duration } \dagger \\
\text { which serum } \\
\text { concs exceed } \\
\text { MIC (days) }\end{array}$ & $\begin{array}{c}\text { Bactericidal } \\
\text { activity }\end{array}$ \\
\hline Rif ampicin & 0.3 & 600 & 30 & 1 & +++ \\
\hline Dapsone & 0.003 & 100 & 500 & 10 & + \\
\hline Acedapsone & $0.003 \S$ & 225 & 15 & 200 & - \\
\hline Ethionamide & $0.05^{\circ}$ & 500 & 60 & 1 & ++ \\
\hline Prothionamide & 0.05 & 500 & 60 & 1 & ++ \\
\hline Thiacetazone & 0.2 & 150 & 8 & 2 & - \\
\hline Sulphamethoxypyridazine $\|$ & 30 & 1000 & 3 & 3 & - \\
\hline Thiambutosine & 0.5 & 1500 & 1 & $<1$ & - \\
\hline
\end{tabular}

* Ratio of peak serum concentration in man after a single dose to MIC determined in the mouse.

† Serum concentrations in man after a single dose.

$\ddagger$ Purely bacteriostatic;,,++++++ , relative degrees of bactericidal activity.

$\S$ Acedapsone is inactive against $M$. leprae but is converted to dapsone-the figures for MIC and peak serum concentration refer to the values for dapsone.

$\|$ Cross-resistant with dapsone.

I Manuf acture discontinued. 
antileprosy activity of the thioamides in patients has yet to be fully evaluated. The cross-resistance demonstrated between the thioamides and thiacetazone indicates that only one drug from this group should be used in combination with other antileprosy drugs.

The role of combinations of drugs for eliminating or decreasing the number of persisting bacilli has yet to be determined. The current THELEP trials should establish the best possible combinations.

\title{
(5) WORKSHOP ON EPIDEMIOLOGY AND CONTROL INCLUDING FIELD THERAPY
}

Chairman:

\author{
S. K. Noordeen
}

Participants: A. Alvaranga; F. Gjalt; V. Ekambaram; J. C. Gatti; J. Languillon; J. Walter; M. F. R. Waters; Unable to attendA. A. Juscenko; W. F. Kirchheimer; M. F. Lechat; T. Meade; D. V. Opromolla Aranjo

\section{EPIDEMIOLOGY}

Only a limited amount of additional information on the epidemiology of leprosy has accumulated since the last Congress, and there is a continuing need for more planned studies on various aspects of the problem. One of the important requirements for field studies is clear and comparable terminology, so that geographical comparisons are possible. There is also a need for more information on mortality rates of leprosy patients and also on spontaneous inactivation of the disease in certain types of leprosy.

One of the major handicaps in the study of the epidemiology of leprosy, particularly on transmission, is the lack of a simple and dependable test to identify subclinical infection in the field, despite the considerable progress which has been made in developing immunological tests. The available information indicates that leprosy is a disease of high infectivity and low pathogenicity. With regard to transmission of the disease, there is more and more evidence of the importance of airborne spread, although other modes of transmission cannot be ruled out. The available evidence on arthropod transmission is inadequate to permit definite conclusions. However, there is less and less justification for insisting on the necessity for direct, prolonged, intimate contact for transmission of the disease. There is also the possibility of a carrier state in leprosy in view of the occurrence of acid-fast-bacilli in the skin and nose of apparently healthy persons, and studies on the occurrence of such bacilli should be repeated in combination with mouse footpad and serological studies. Regarding possible extra-human reservoirs of infection, it is difficult to evaluate the significance of the occurrence of leprosy or leprosy-like disease in armadillos in certain parts of the U.S.A., and it may be worthwhile to look for similar reservoirs in other parts of the world, using modern methods.

The role of genetic predisposition in leprosy is not clear in view of the inadequacy of the available information. Further precise studies on the importance of genetics in leprosy are indicated. 
One area of research in leprosy that could be fruitful is "risk factor" studies, where association of leprosy with certain variables related to environment or host could be studied prospectively with the hope that it may be possible to contribute to disease prevention through intervention or manipulation of the risk factors identified.

\section{LEPROSY CONTROL}

In leprosy control, programme planning is vital for successful control work. Programme formulation provides a logical process to ensure a full analysis of the current epidemiological, operational, and managerial problems. Such a formulation requires specially trained staff. A programme should include precise objectives, targets, manpower requirements, physical facilities, financial resources, timings of activities and their interrelations. Programming incorporates implementation and evaluation; the latter should include specification of the subject, verification and analysis of information support, review of original programme planning, and review of progress on (a) operational efficiency, such as staff competence, case detection activities, case holding, bacteriological monitoring, etc; (b) epidemiological effectiveness, such as reduction of prevalence and incidence rates and of def ormity rates.

The programme planning should lead to a gradual integration of specialized programmes into the general and primary health services, which should eventually take full responsibility. This is a task for which the general health workers must be trained and motivated in order to prevent failures.

For the analysis of the leprosy situation and to make the comparison between data from different regions possible, it is recommended that a uniform leprosy information system be developed.

Case detection methods should, among others, include methods to encourage voluntary reporting through health education of the community utilizing, among others, mass media such as radio, TV, etc. In urban areas, motivation of general practitioners could be useful for case detection; they should coordinate with health authorities. Where facilities are available, lepromin testing is useful for identifying early cases which are lepromin negative.

With regard to release of patients from control, the recommendations of the Fif th WHO Expert Committee on Leprosy were considered to be valid. However, further studies on optimal duration of chemotherapy of paucibacillary leprosy were considered necessary.

Regarding treatment delivery, while the mode of treatment delivery will depend upon individual situations, it is necessary to carry out periodic checks on drug intake through urine tests. Every effort should be made to maintain regularity of treatment of patients, particularly of patients with multibacillary leprosy. In this connection it is necessary to ensure high standards in patient care.

Regarding primary prevention, although studies on chemoprophylaxis have established their moderate protective value, the application of chemoprophylaxis on a mass scale will not be practicable. Notwithstanding the difficulties, chemoprophylaxis can still be recommended in individual 
situations, where the risk of contracting leprosy is considered to be unduly high. Considering the limited prophylactic value of $\mathrm{BCG}$, there is a need to develop a specific antileprosy vaccine.

\section{FIELD THERAPY}

The Workshop reviewed field therapy of leprosy in the light of the recent advances in the pharmacology of the antileprosy drugs, the increasing incidence of dapsone resistance reported from different parts of the world, and the need for effective, acceptable, practicable, simple and supervisable therapy, both for infection with Mycobacterium leprae and for the treatment of reactions.

The group reviewed the drugs available, and confirmed the importance of using bactericidal drugs, namely dapsone in full dosage, rifampicin, clofazimine and ethionamide or prothionamide.

Dapsone remains the basis of treatment, at a dosage of 6 to $10 \mathrm{mg}$ per $\mathrm{kg}$ body weight per week. The drug should be commenced in full dosage, without any initial "build-up", and this dosage should be kept unchanged throughout treatment, and should not be interrupted or altered during reactions.

In tuberculoid (TT and BT), borderline (BB) and indeterminate (I) leprosy, monotherapy with dapsone is acceptable. In lepromatous ( $L L$ and BL) leprosy, it is recommended that an initial intensive phase of combined therapy should be given.

Recommended adult regimens for the treatment of lepromatous leprosy include:

(a) rifampicin $600 \mathrm{mg}$ daily for a minimum of 2 weeks, plus dapsone 100 mg daily, indefinitely;

(b) rifampicin $1500 \mathrm{mg}$ in a single dose on the first day of treatment, plus dapsone $100 \mathrm{mg}$ daily, indefinitely;

(c) clofazimine $100 \mathrm{mg}$ daily for 2 months, then $100 \mathrm{mg}$ three times a week for 4 months, plus dapsone $100 \mathrm{mg}$ daily, indefinitely;

(d) ethionamide $375 \mathrm{mg}$ daily for 3 months, plus dapsone $100 \mathrm{mg}$ daily, indefinitely.

Pre-programme studies of ethionamide are recommended in any area, before the drug is introduced generally.

Choice of regimen depends upon cost, cultural acceptance and toxicity. If none of the above regimens can be afforded, the alternatives are:

(e) thiacetazone $150 \mathrm{mg}$ daily for 1 year, plus dapsone $100 \mathrm{mg}$ daily;

(Thiacetazone may have an unacceptably high incidence of toxic sideeffects, especially in East Asia; its cost over 1 year equals that of a single dose of rifampicin, and thiacetazone-resistant $M$. leprae show cross-resistance with ethionamide.)

(f) dapsone $100 \mathrm{mg}$ daily indefinitely, ensuring regularity of treatment. 


\section{Dapsone resistance}

Secondary dapsone resistance has to date only been reported in lepromatous ( $\mathrm{LL}$ and $\mathrm{BL}$ ) leprosy. The diagnosis should be confirmed by a medical officer. It is appreciated that mouse footpad confirmation is not widely available. However, random specimens of the patient's urine should normally be tested to confirm the presence of dapsone. Further information is needed on the incidence and prevalence of dapsone-resistant leprosy in different geographical areas.

Recommended adult regimens for the treatment of dapsone-resistant patients include:

(a) rifampicin $600 \mathrm{mg}$ daily for $1 \mathrm{month}$, plus clofazimine $100 \mathrm{mg}$ daily for 6 months, then $100 \mathrm{mg}$ three times a week indefinitely;

(b) rifampicin $600 \mathrm{mg}$ daily for $1 \mathrm{month}$, plus ethionamide $375 \mathrm{mg}$ daily, indefinitely;

(c) ethionamide $375 \mathrm{mg}$ daily for 3 months, plus clofazimine $100 \mathrm{mg}$ daily for 6 months, then $100 \mathrm{mg}$ three times a week indefinitely;

(d) clofazimine $100 \mathrm{mg}$ daily for 6 months, plus ethionamide $375 \mathrm{mg}$ daily indefinitely.

Primary dapsone resistance may occur in any type of leprosy, and this possibility should always be kept in mind, especially among contacts of known cases of secondary resistance.

\section{Field therapy of reactions}

Effective treatment of reactions is essential for the well-being of patients, and to retain their cooperation with drug therapy. This involves improved training of field staff, and the provision of referral centres for the treatment of severe reactions. Mild reactions should be treated in the field, the patient being seen regularly; patients suffering from severe reactions should be sent immediately to the referral centre, antileprosy treatment being continued unchanged.

\section{Prevention of deformities}

Prevention of deformities depends on the early diagnosis of leprosy, eff ective antileprosy treatment, effective treatment of reactions to prevent (further) nerve damage, and education of the patients in the care of anaesthetic limbs.

\section{WORKSHOP ON HUMAN ASPECTS IN THE TREATMENT OF LEPROSY PATIENTS}

Chairman: $\quad$ Mr Marcel Farine

Rapporteur: Dr Ernest Fritschi

Participants: Dr Gilberto Rodriguez Ochoa; Sr Gebre Mariam Senkenesh; Dr (Mrs) Turkan Saylan; Dr V. P. Macaden; Mr R. S. Mani; Mr D. Von der Weid; Dr (Miss) Grace Warren; Mrs Alicia E. Kaufmann de Swiec; Rev. Rocco Serra; Mr Iman Bijleveld 
Observers: $\quad$ Mr A. D. Askew; Dr L. M. Baliña (in place of Dr Manuel M. Gimenez)

\section{INTRODUCTION}

Mass treatment and case-finding in any endemic area tends to have the effect of dehumanising the individual patient. Therefore, each member of the team, medical, paramedical and social, while bringing to bear on the patient his own particular expertise, must constantly remind himself of the patient's total needs - physical, emotional and social, and treat him as a fellow human being. The effective moments for the application of the human element to the treatment of leprosy patients are during the first contacts of the team with the patient.

\section{GENERAL CONSIDERATIONS}

The extent of the trauma inflicted on the patient by the diagnosis of leprosy is very important. The word "leprosy" is to be used with caution, since it tends to have a socio-historical, in addition to a medical connotation.

The stigma attached to leprosy can only be overcome by a continuous programme of health education at all levels and for all target audiences. The shock of the first diagnosis should be communicated to the patient with kindness. The patient often may not believe it until the deformities appear. This lack of acceptance of the diagnosis is a significant factor, along with socioeconomic considerations, in chronic absenteeism from treatment by the patient.

\section{CASE FINDING AND CASE HOLDING}

Finding patients in the community and holding their confidence during treatment is a continuing concern. The whole team must be imbued with an attitude of sympathy for the patient. His privacy must be respected and his identity preserved. The method of door to door delivery of medication, and unscheduled follow-up visits to ensure that the patient is taking his tablets has proved useful.

There are many factors which render regular clinic attendance difficult for the patient, and the health education team should take account of these problems in their presentation.

The continuing health education of the patient, his family and society should be accepted as requiring the highest priority.

Well qualified social workers with carefully defined job descriptions must be engaged. They should ensure that the other members of the team are trained, not merely in the detection of early lesions and medical aspects, but in psychology, techniques of communication and counselling.

\section{CENTRES FOR PATIENT CARE}

The old pattern of institutional care has largely given place to field clinics. Institutions however, still remain a necessity. The nature of the institution is important, and the difference in the psychological impact on patients in a leprosarium and a hospital is considerable. Emphasis must be given to the need 
for understanding the patient's emotional reactions, and in the hospital set-up, the staff must take time to care for these too.

It is important to establish an interdisciplinary team participation in planning the treatment of the patient.

Hospitalization should be short-term and interrupted. Each admission should be for a specific and documented objective, such as the healing of an ulcer, operation of a hand, treatment of neuritis, etc., the patient being discharged after this target is achieved, to be re-admitted later for another reason if necessary. Patients should be taught self-care of their hands and feet at home.

\section{REHABILITATION AND RE-INTEGRATION}

In relation to this subject, health education was again the line strongly advocated by all the participants. The need was stressed to implement the Havana Congress' (1948) decision to educate all levels: the medical profession, politicians and the State administration, schools and society at large, of which of course the patient is also a member. The central place of domiciliary rehabilitation has to be emphasized and the team approach is required to keep the patient in his home environment.

The beggar presents a real problem of socio-economic failure in developing countries. Rehabilitation of these patients is difficult but not impossible. However, efforts and resources should also be used to prevent patients from descending to this level.

Rehabilitation and re-integration of the patient in society, especially if he has deformities, can only be achieved by sustained efforts on the part of the patient, the medical, paramedical and social team, and society as a whole. It is necessary to discover and harness the patient's abilities and not be distracted by his disabilities.

Rehabilitation should be planned after careful consideration as to whether further displacement is essential or not. All measures taken for persons disabled because of leprosy should be in the context of resolutions already adopted by ECOSOC, WHO, and ILO (see reference). Care must be taken not to make leprosy a qualification for special privileges.

It has also been proved that it is less expensive to provide appropriate rehabilitation services than to provide the necessary care for an individual who, without rehabilitation, depends on public help.

\section{BASIC PRINCIPLES FOR THE HUMANE TREATMENT OF LEPROSY PATIENTS}

(1) Health education, both general and specific, must be the basis of all rehabilitation.

(2) A natural and empathetic approach to the patient is required to reduce psychic trauma and loss of identity to the minimum.

(3) Hospitalization should be for short periods, each with definite objectives. Long-term treatment should be interrupted by periods at home from time to time, to avoid long separations from the family. 
(4) Much time should be spent by the team in instructing the patient in the care of his hands and feet and the prevention of injuries.

(5) The approach to rehabilitation should not be confined to one channel, such as sheltered industry or domiciliary employment, but all efforts should be made to find out the type of rehabilitation must suited to the country, the environment, the patient's aptitude, skills and social status, and the available funds for capital costs per work place.

\section{Recommendations}

To encourage further investigation and to carry out the above basic principles (see para.6), it is particularly necessary:

(a) To organize a research centre and/or referral library, to collect a bibliography of all publications on social aspects of leprosy patient care, and to make these available to those interested.

(b) To inform on a continuing basis and with discernment, every section of the population, especially in areas and countries where leprosy is endemic, utilizing mass media about the leprosy problem and its social aspects, where possible in the context of general health education, in order to facilitate the acceptance of leprosy patients.

(c) To produce articles, TV and radio features, as well as booklets in different languages on the human aspects of leprosy.

Wherever false concepts are being propagated in the mass media, the concerned organizations must contact the authors, request the withdrawal of any misleading ideas and offer in their place the accepted facts of the disease. If necessary a public protest may be made, utilizing the same media.

(d) To organize in endemic areas and countries regular courses in schools, universities, medical schools, etc., not only on the medical aspects of the disease, but also on its historical, social and economic aspects, pointing out the necessity for humane treatment of the patients.

(e) To train in social aspects (by courses, conferences, seminars, etc.) the personnel engaged in case-finding and working in health centres, hospitals or other services, and to engage qualified social workers, especially for important projects.

(f) To bring to the notice of everybody concerned (especially social, medical, commercial and pharmaceutical agencies) these important aspects of leprosy programmes; in particular, to encourage investment in research and development, and lower prices of anti-leprosy drugs.

(g) To awake the conscience of governments to provide all facilities for the production of anti-leprosy drugs and equipment, and where necessary to waive customs duties and other formalities connected with the import of these drugs and equipment.

(h) To draw the attention of intergovernmental organizations such as UNO, WHO and ILO, international non-governmental organizations such as ILA and ILEP, as well as governments and private organizations, to the need for a concern for the social and human aspects in the treatment of 
leprosy patients, as outlined in this report, taking into consideration recommendations and guidelines already adopted. To achieve these objectives, it is essential that all these agencies act together in close cooperation.

\section{Reference}

No. 99 of ILO (1955).

No. A29.68 of WHO (1976).

No. 1921 (LVIII) of ECOSOC (1975).

\section{(7) WORKSHOP ON TEACHING MATERIAL FOR LEPROSY WORKERS}

Chairman: Dr W. Felton Ross

Rapporteurs: Miss Jane Neville; Dr Ken Seal; Dr B. Landheer

Participants: Dr Joe J. Arvelo; Dr M. Aschhoff; Dr Wanda Blenska; Dr M. Bourges; Dr J. A. Cap; Med Général A. Carayon; Dr C. J. G. Chacko; Dr D. S. Chaudhury; Dr N. Desikan; Mrs Soledad S. Griño; Dr E. D. Kelly; Dr Do Il Kim; Mr Herman Kober; Dr A. C. McDougall; Dr Sandy Ritung; Med Général P. B. Saint Andre; Dr H. W. Wheate; Dr Yo Yuasa; Dr J. A. K. Clezy; Dr Schaller; Dr Roy E. Pfaltzgraff

\section{Purposes}

(1) To review currently available teaching material in the leprosy field.

(2) To identify the main training needs, not met by existing material.

(3) To develop plans and coordinate efforts to meet those needs.

\section{INTRODUCTION}

The production and distribution of suitable teaching/learning material in leprosy is of increasing importance as:

(a) more and more states adopt the principles of community health and widen the range of health personnel engaged in leprosy patient care and leprosy control;

(b) the technologies of leprosy patient care, leprosy control and health programme management become more advanced;

(c) new developments in education offer the possibilities of greatly increased effectiveness in teaching.

\section{PROCEDURE}

Members of the workshop brought copies of over 100 different pieces of teaching material in a number of languages. 
As a basis for reviewing this material, the workshop adopted the following categories of tasks in leprosy work: The Public, Patients, Health Work I, Health Work II, Health Work III and Specialists (see Annex). Limited by time, members of the workshop concentrated on material for Health Work I, II and III. The members divided into groups to review the literature, using a literature assessment form, a copy of which is attached.

Pieces of literature were reviewed by up to six participants.

\section{CONCLUSIONS}

(a) A great deal more teaching material exists than is generally known and available.

(b) There are still parts of the world where shortage of currency limits the availability of teaching material.

(c) Serious needs for more material exist in the following areas:

(i) basic leprosy for village level workers;

(ii) supervision;

(iii) programme management;

(iv) laboratory technology;

(v) a periodical for middle level workers to fill the gap existing between the magazine "Partners" and "Leprosy Review".

\section{Recommendations and Actions}

\section{ADVERTISEMENT}

(1) In order to begin to make existing literature more widely known, members of the workshop agreed to:

(a) collate and publish the list of material available at the workshop;

(b) prepare an annotated bibliography of literature reviewed at the workshop;

(c) prepare more complete bibliographies in French, Spanish and English.

(2) The workshop welcomed the steps taken by The Royal Tropical Institute, Amsterdam, to develop a leprosy information service which will collect and register and provide information about literature and audio-visual aids available in the leprosy field.

(3) The workshop recommends that all who publish teaching material in leprosy send sample copies to The Royal Tropical Institute, Mauritskade 63, Amsterdam-O, Netherlands.

(4) The workshop recommends that a series of teaching material- resourceand display-centres be established in major endemic areas and in the main leprosy training centres. 


\section{DISTRIBUTION}

(1) The workshop recommends that existing distribution centres make their services more widely known.

(2) The workshop recommends that a far greater priority in budgeting be given to literature, including periodicals, by all the agencies involved in leprosy programmes and that agencies willing to provide funds for literature should make this fact known.

6. PUBLICATION

(1) Authors seeking to produce material for teaching need the help of experts in design and layout.

A workshop was proposed with the object of providing a manual for guidance in this field.

(2) Field trials for the evaluation of materials are recommended.

(3) There is a need for a critical appraisal of the leprosy component within general medical text books.

\section{WORKSHOPS}

(1) It is recommended that a workshop on educational technology be held at the next Leprosy Congress.

(2) Audiovisual aids were not reviewed and in view of rapid developments in this field this could also be the subject of a workshop.

\section{ANNEX}

Roles in leprosy control

\begin{tabular}{ll}
\hline Category & Functions \\
\hline The Public & Accept and support patients \\
& Recognize leprosy \\
Seek medical advice & Recognize leprosy \\
Patients & Seek medical advice \\
& Take treatment \\
& Practice self-care \\
Health Worker Category I & Teach and treat leprosy patients \\
& Recognize complications \\
& Recognize leprosy \\
& Keep records \\
Health Worker Category II & Diagnose and classify leprosy and deformity \\
& Recognize complications \\
& Teach patients self-care \\
Teach the public \\
Teach health worker I \\
Keep records \\
\\
Health Worker Category III & Diagnose complications \\
& Manage complications \\
& Teach health worker II \\
& Keep records \\
\hline
\end{tabular}




\section{BOOK ASSESSMENT FORM}

TITLE . . .

PUBLISHER

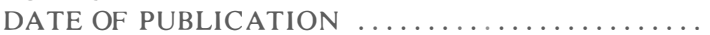

LANGUAGE - French ( ), Spanish ( ), Portuguese ( ), German ( ), English ( ), Other

TO BE USED BY:

Job Title

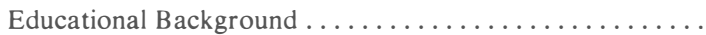

FOR WHAT PURPOSE:

General Introduction $\ldots \ldots \ldots \ldots \ldots \ldots \ldots \ldots \ldots$

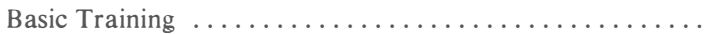

Advanced Training

* Suitable; ${ }^{* *}$ Very suitable; ${ }^{* * *}$ Outstanding

— Not Suitable

AUTHOR

PRICE (in U.S.\$)

PAGE SIZE

BINDING - PAPER, CLOTH, HARD

EDITION NUMBER

METHOD OF USE:

For Self Instruction

Part of a Course . .

A Working Manual

\section{CONTENT:}

\section{General}

1. Are the objectives stated?

2. Is it accurate and up-to-date?

3. Does it contain sufficient real examples . for local application?

4. Does it contain sufficient practical instructions?

5. Does it include sufficient accurate summaries?

Specific to user and purpose given above-

6. Does it include sufficient relevant review questions?

7. Is the subject matter relevant?

8. Is the level of difficulty appropriate?

9. Is the coverage appropriately complete?
( ) In each chapter

( ) Entirely

( ) Yes

( ) Yes

( ) Yes

( ) Yes

( ) Completely

( ) Yes

( ) Yes
( ) Elsewhere

( ) Mainly

( ) Too many

( ) Too many

( ) Too many

( ) Too many

( ) Mainly

( ) Too easy

( ) Too much is included
( ) Not at all

( ) Serious errors

( ) Too few

( ) Too few

( ) Too few

( ) Too few

( ) Partly

( ) Too difficult

( ) Serious omissions

10. Other comments:

\section{PRESENTATION:}

1 . Is the type easy to read?

2. Is the line length appropriate?

3. Are illustrations relevant?

4. Are illustrations annotated?
( ) Yes

( ) Yes

( ) Yes

( ) Yes
( ) Too large \& spread out

( ) Too long

( ) Confusing

( ) Too much
( ) Too small \& cramped

( ) Too short

( ) No

( ) Too little 

5. Are illustrations of good quality?
( ) Yes
( ) To some
( ) No
6. Language - Vocabulary
( ) Appropriate
( ) Too
difficult
7. Language-Structure
( ) Appropriate
( ) Too
complex
( ) Too easy
( ) Too simple

Which of the following additional learning aids are used: Abstracts, algorithms, boxed slogans, cartoons, case studies, decision tables, diagrams, exercises, footnotes, graphs, lists, references, sense lines, tables.

Please circle and comment as to quantity, relevance, and quality of the aids used.

OVERALL ASSESSMENT

FOR USE BY:

\begin{tabular}{lll}
\hline Category & Purpose & Rating \\
\hline
\end{tabular}

Public

Patients

H. Worker 1

H. Worker 2

H. Worker 3

Specialist

Physical Th'y

H. Educator

Social W.

\begin{tabular}{|c|c|c|c|c|c|c|}
\hline Purpose & $\begin{array}{l}\text { Introduction } \\
\text { Basic Training } \\
\text { Advanced Training }\end{array}$ & $\begin{array}{l}1 \\
2 \\
3\end{array}$ & $\mathrm{R}$ ating & $\begin{array}{l}\text { Outstanding } \\
\text { Very Suitable } \\
\text { Suitable }\end{array}$ & $\begin{array}{l}* * * \\
* * \\
*\end{array}$ & $\begin{array}{l}\text { H. Worker } 1 \bumpeq \text { Health Worker } \\
\text { H. Worker } 2 \bumpeq \text { Lepr. Auxiliary } \\
\text { H. Worker } 3 \bumpeq \text { Doctor } \\
\text { Approximate } \\
\text { Equivalents }\end{array}$ \\
\hline
\end{tabular}

Applicability

Relative rating

Summary
- Useful only in country of origin Wide usefulness in original language I recommend translation into

-Nothing else exists in its field Better than Not as good as

-Fills a gap in the literature Needs revision \& updating Should be replaced by something better Define "something better" below
( )

( )

( )

( )

This book contains sufficient material to enable the reader to:

\begin{tabular}{l}
\hline No Yes \\
\hline 1. Accept/Support patients \\
2. Recognize leprosy \\
3. Give out treatment \\
4. Recognize complications \\
5. Diagnose leprosy \\
6. Classify leprosy* \\
7. Grade deformities* \\
8. Manage leprosy \\
9. Diagnose complications \\
10. Manage complications
\end{tabular}

* Name system used. 